\title{
Continuous-variable quantum process tomography with squeezed-state probes
}

\author{
Jaromír Fiurášek \\ Department of Optics, Palacký University, 17. listopadu 1192/12, 77146 Olomouc, Czech Republic
}

\begin{abstract}
We propose a procedure for tomographic characterization of continuous variable quantum operations which employs homodyne detection and single-mode squeezed probe states with a fixed degree of squeezing and anti-squeezing and a variable displacement and orientation of squeezing ellipse. Density matrix elements of a quantum process matrix in Fock basis can be estimated by averaging well behaved pattern functions over the homodyne data. We show that this approach can be straightforwardly extended to characterization of quantum measurement devices. The probe states can be mixed, which makes the proposed procedure feasible with current technology.
\end{abstract}

PACS numbers: $03.65 . \mathrm{Wj}$

\section{INTRODUCTION}

As the complexity of quantum information processing devices increases, there is a growing need for tools for their characterization and benchmarking. Quantum operations and channels can be completely characterized by quantum process tomography [1 5], which represents an extension of quantum state tomography 6 , to quantum operations. Typically, the quantum operation $\mathcal{E}$ is probed with a sufficient number of input states $\rho_{j}$, measurements in several different bases are performed on the output states $\mathcal{E}\left(\rho_{j}\right)$, and the quantum operation is reconstructed from the experimental data. Alternatively, in the ancilla-assisted quantum process tomography 9.13 . the operation $\mathcal{E}$ is probed with one part of a single fixed entangled bipartite state $\rho_{A B}$ and $\mathcal{E}$ is determined from measurements on the output bipartite state. This latter approach is based on the Choi-Jamiolkowski isomorphism [14, 15], which tells us that if the probe state is pure and maximally entangled, $|\Phi\rangle_{A B}=\frac{1}{\sqrt{d}} \sum_{j=0}^{d-1}|j j\rangle_{A B}$, then the output bipartite state $\chi_{A B}=\mathcal{I}_{A} \otimes \mathcal{E}_{B}(|\Phi\rangle\langle\Phi|)$ is directly isomorphic to the operation $\mathcal{E}$. Here $d$ denotes the dimension of input Hilbert space $\mathcal{H}_{\text {in }}$ and the states $|j\rangle$ form an orthonormal basis in $\mathcal{H}_{\text {in }}$.

Quantum process tomography works particularly well for few-qubit systems, and it has been successfully applied in the past to characterization of various singlequbit and two-qubit operations [11, 12, 16, 21]. As the number of qubits $N$ increases, the full tomography becomes challenging since the number of parameters that have to be estimated grows exponentially with $N$. In some cases, scalable quantum process reconstruction may be achieved e.g. by approximating the operator $\chi$ by a matrix product state [22, 23] or by using compressed sensing techniques $24-26]$.

Besides the issue of Hilbert space dimension, the quantum process tomography is also affected by the range of practically accessible input probe states. This is particularly relevant for continuous variable quantum process tomography [3, 4, 27, 30], which aims at characterization of quantum operations on modes of quantized electromagnetic fields. Here, the most natural and readily available probe states are represented by coherent states $|\alpha\rangle$, and the output states can be conveniently measured with homodyne detectors [6, 8]. Recently, this approach has been successfully employed to characterize a single-mode lossy channel 27, and a conditional single-photon addition and subtraction [30. Moreover, the coherent states were also used as probes for complete tomographic characterization of single-photon detectors 31 35. Probing quantum processes with coherent states essentially amounts to determining a Husimi $Q$-function of the operator $\chi$. More precisely, assuming that the measurements on output states are described by a POVM with elements $\Pi_{j}$, the probability of measurement outcome $\Pi_{j}$ for input probe coherent state $|\alpha\rangle$ reads $p_{j}(\alpha)=\operatorname{Tr}\left[\left|\alpha^{*}\right\rangle\left\langle\alpha^{*}\right| \otimes \Pi_{j} \chi\right]$. Usually, one would like to reconstruct the matrix elements of $\chi$ in Fock basis. To see the connection between the Husimi $Q$-function and the matrix elements in Fock basis, recall that the $Q$-function of an operator $A$ is defined as $Q(\alpha)=\langle\alpha|A| \alpha\rangle / \pi$. We have

$$
Q\left(\alpha, \alpha^{*}\right)=\frac{e^{-|\alpha|^{2}}}{\pi} \sum_{m, n=0}^{\infty} \frac{\alpha^{* m} \alpha^{n}}{\sqrt{m ! n !}} A_{m, n}
$$

which shows that the $Q$-function is a generating function of matrix elements $A_{m, n}=\langle m|A| n\rangle$ in Fock basis [28],

$$
A_{m, n}=\left.\frac{\pi}{\sqrt{m ! n !}} \frac{\partial^{m+n}}{\partial \alpha^{* m} \partial \alpha^{n}}\left[Q\left(\alpha, \alpha^{*}\right) e^{|\alpha|^{2}}\right]\right|_{\alpha=\alpha^{*}=0}
$$

Here $\alpha$ and $\alpha^{*}$ are formally treated as independent variables. Estimation of $A_{m, n}$ from experimental data requires inversion of Eq. (1) when the $Q$-function is not known precisely. This can be a delicate procedure sensitive to statistical fluctuations of the data.

In Ref. [27], elements of quantum process matrix of a lossy channel were reconstructed from the experimental data with the help of regularized version of GlauberSudarshan $P$-functions [36] of operators $|m\rangle\langle n|$. This approach approximates the calculation of derivatives in Eq. (2) by evaluation of a suitable linear combination of the experimental data. In Ref. [31], POVM elements of a single-photon detector were reconstructed from measurements on probe coherent states by solving a convex optimization problem that included an extra constraint 
which ensured a smooth structure of the reconstructed POVM elements. Later on, maximum-likelihood estimation was employed for reconstruction of quantum operations and detectors probed with coherent states 30, 33. This latter approach avoids the complications with direct linear inversion (2), but it requires some truncation of the infinite-dimensional operator $\chi$.

In this paper, we investigate characterization of continuous variable quantum operations which is based on single-mode squeezed probe states and homodyne detection on output states. By using squeezed states instead of coherent states we avoid the problems with linear inversion of the data and we show that the matrix elements of quantum process $\chi$ in Fock basis can be determined by averaging suitable well behaved pattern functions $37-$ 39] over the homodyne data. Our procedure assumes that all probe states have the same variances of squeezed and anti-squeezed quadratures, and these variances need to be known and kept constant during the whole measurement. The probe states also need to be phase shifted and coherently displaced in a controlled way, which is feasible with current technology. Importantly, our procedure works for realistic mixed squeezed states and the only requirement is that the variance of the squeezed quadrature is below the coherent state level. Although we focus on linear reconstruction based on the formalism of pattern functions, the data could be processed by other means, such as the maximum likelihood estimation. Our work provides an important insight into the utility of squeezed states for tomography of quantum processes.

\section{QUANTUM PROCESS TOMOGRAPHY}

In what follows we shall consider chracterization of a single-mode quantum operation $\mathcal{E}$. According to the Choi-Jamiolkowski isomorphism [14, 15], such operation can be represented by a positive semidefinite operator $\chi$ on a Hilbert space of two modes,

$$
\chi=\mathcal{I} \otimes \mathcal{E}(\Psi),
$$

where $\mathcal{I}$ stands for the identity channel and $\Psi=|\Psi\rangle\langle\Psi|$ denotes a density matrix of an infinitely squeezed EPR state,

$$
|\Psi\rangle=\sum_{n=0}^{\infty}|n n\rangle
$$

The input-output transformation $\rho_{\text {out }}=\mathcal{E}\left(\rho_{\text {in }}\right)$ can be expressed as

$$
\rho_{\text {out }}=\operatorname{Tr}_{\text {in }}\left[\rho_{\text {in }}^{T} \otimes I \chi\right],
$$

where $T$ denotes transposition in Fock basis, $I$ stands for the identity operator, and $\operatorname{Tr}_{\text {in }}$ denotes partial trace over the input mode. In Fock basis, the formula (5) explicitly reads,

$$
\rho_{\mathrm{out}, m, n}=\sum_{k=0}^{\infty} \sum_{l=0}^{\infty} \chi_{k m, l n} \rho_{\mathrm{in}, k, l} .
$$

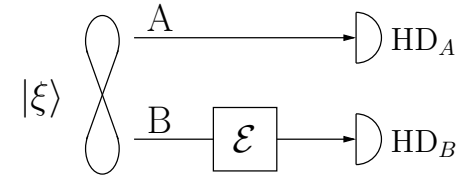

(a)
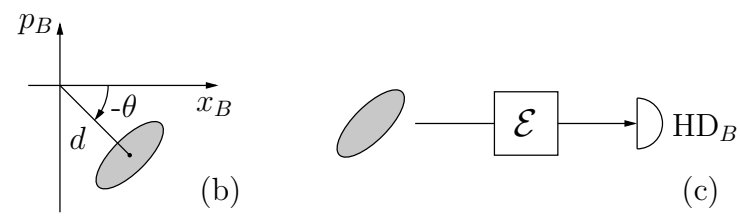

(b)

FIG. 1: (a) Ancilla-assisted quantum process tomography [3, 9, 10] of a single-mode operation $\mathcal{E}$. The operation is applied to one part of input two-mode squeezed vacuum state $|\xi\rangle$ and both output modes are measured with balanced homodyne detectors $\mathrm{HD}_{A}$ and $\mathrm{HD}_{B}$. (b) Homodyne measurement of quadrature $x_{A}^{\theta}$ of mode A of the two-mode squeezed vacuum state $|\xi\rangle$ prepares mode B in a coherently displaced and rotated Gaussian squeezed state. (c) The ancilla assisted tomography is therefore equivalent to probing the operation $\mathcal{E}$ with a suitably chosen ensemble of single-mode squeezed states.

Here $\rho_{m, n}=\langle m|\rho| n\rangle$ and $\chi_{k m, l n}=\langle k m|\chi| l n\rangle$. Identity channel $\mathcal{I}$ is isomorphic to the EPR state [4], $\chi_{\mathcal{I}}=\Psi$, and $\chi_{\mathcal{I}, k m, l n}=\delta_{k m} \delta_{l n}$.

The input-output transformation (5) can be also formulated for phase-space representations. Let $W_{\text {in }}(x, p)$ and $W_{\text {out }}(x, p)$ denote the Wigner functions of input and output density operators $\rho_{\text {in }}$ and $\rho_{\text {out }}$, respectively, and let $W_{\chi}\left(x_{\text {in }}, p_{\text {in }}, x_{\text {out }}, p_{\text {out }}\right)$ denote the Wigner function of operator $\chi$. The partial trace (5) can be rewritten as an integral over the phase space of the input mode,

$$
\begin{aligned}
W_{\text {out }}\left(x_{\text {out }}, p_{\text {out }}\right)= & 2 \pi \int_{-\infty}^{\infty} \int_{-\infty}^{\infty} W_{\text {in }}\left(x_{\text {in }},-p_{\text {in }}\right) \\
& \times W_{\chi}\left(x_{\text {in }}, p_{\text {in }}, x_{\text {out }}, p_{\text {out }}\right) d x_{\text {in }} d p_{\text {in }} .
\end{aligned}
$$

where $W_{\text {in }}\left(x_{\text {in }},-p_{\text {in }}\right)$ is a Wigner function of the transposed input state $\rho_{\text {in }}^{T}$.

Our goal is to establish a procedure for determination of the matrix elements $\chi_{k m, l n}$ from experimental data. Formula (3) suggests that this could be achieved by probing the quantum operation $\mathcal{E}$ with one part of the EPR state $|\Psi\rangle$. To make this continuous-variable ancilla-assisted quantum process tomography [9, 10] experimentally feasible, the unphysical infinitely squeezed EPR state may be replaced with a two-mode squeezed vacuum with finite squeezing [3],

$$
|\xi\rangle=\sqrt{1-\lambda^{2}} \sum_{n=0}^{\infty} \lambda^{n}|n n\rangle,
$$

where $\lambda=\tanh r$, and $r$ denotes the squeezing constant. After some algebra, we find that the elements of the quantum process matrix $\chi$ can be determined as properly rescaled elements of the output two-mode state 
$\sigma^{\lambda}=\mathcal{I} \otimes \mathcal{E}(\xi)$, where $\xi=|\xi\rangle\langle\xi|$,

$$
\chi_{k m, l n}=\left(1-\lambda^{2}\right)^{-1} \lambda^{-(k+l)} \sigma_{k m, l n}^{\lambda} .
$$

If both modes of the output state $\sigma^{\lambda}$ would be measured with homodyne detectors, see Fig. 1(a), then the matrix elements $\sigma_{k m, l n}^{\lambda}$ could be reconstructed by quantum homodyne tomography [6, 8. Let $\eta_{A}$ and $\eta_{B}$ denote the overall detection efficiency of balanced homodyne detectors $\mathrm{HD}_{A}$ and $\mathrm{HD}_{B}$, respectively. A detector with efficiency $\eta$ can be modeled as a lossy channel with transmittance $\eta$ followed by an ideal detector with unit efficiency. The detectors measure rotated quadratures of modes $\mathrm{A}$ and $\mathrm{B}$, which are specified by angles $\theta$ and $\phi$, respectively,

$$
\begin{aligned}
& x_{A}^{\theta}=\sqrt{\eta_{A}}\left(x_{A} \cos \theta+p_{A} \sin \theta\right)+\sqrt{1-\eta_{A}} x_{A, \mathrm{vac}}^{\theta}, \\
& x_{B}^{\phi}=\sqrt{\eta_{B}}\left(x_{B} \cos \phi+p_{B} \sin \phi\right)+\sqrt{1-\eta_{B}} x_{B, \mathrm{vac}}^{\phi} .
\end{aligned}
$$

Here $x_{J}$ and $p_{J}$ denote the amplitude and phase quadratures of mode $J,\left[x_{J}, p_{K}\right]=i \delta_{J K}$, and $x_{A \text {,vac }}^{\theta}$ and $x_{B \text {,vac }}^{\phi}$ represent quadratures of auxiliary vacuum modes. This measurement samples the joint quadrature distribution $P\left(x_{A}^{\theta}, x_{B}^{\phi} ; \theta, \eta_{A}, \phi, \eta_{B}\right)$ and the matrix elements $\chi_{k m, l n}$ can be determined by averaging the so-called pattern functions over the quadrature statistics [37 39],

$$
\chi_{k m, l n}=\frac{\left(1-\lambda^{2}\right)^{-1}}{4 \pi^{2} \lambda^{k+l}} \int_{-\infty}^{\infty} \int_{0}^{2 \pi} \int_{-\infty}^{\infty} \int_{0}^{2 \pi} P\left(x_{A}^{\theta}, x_{B}^{\phi} ; \theta, \eta_{A}, \phi, \eta_{B}\right) f_{k, l}\left(x_{A}^{\theta}, \eta_{A}\right) f_{m, n}\left(x_{B}^{\phi}, \eta_{B}\right) e^{i(k-l) \theta} e^{i(m-n) \phi} d x_{A}^{\theta} d \theta d x_{B}^{\phi} d \phi .
$$

Here $f_{m, n}(x, \eta)$ represent the loss-compensating singlemode pattern functions for density matrix elements in Fock basis. Explicit analytical expressions for $f_{m, n}(x, \eta)$ are provided in Ref. [39]. Since these expressions are rather cumbersome, we do not reproduce them here. We only note that the pattern functions $f_{m, n}(x, \eta)$ are well defined for $\eta>\frac{1}{2}$ and they diverge when $\eta \rightarrow \frac{1}{2}$.

\section{SINGLE-MODE PROBE STATES}

In this section, we will propose a procedure for quantum process tomography with single-mode squeezed probe states. In particular, we will exploit the fact that the ancilla assisted process tomography with a two-mode squeezed vacuum state $|\xi\rangle$ and individual single-mode homodyne measurements on the output modes is equivalent to preparation of a specific ensemble of displaced and rotated single-mode squeezed states of mode B, followed by probing the quantum operation $\mathcal{E}$ with these states 23 . To see this equivalence, we rewrite the joint quadrature distribution as

$$
\begin{aligned}
P\left(x_{A}^{\theta}, x_{B}^{\phi} ; \theta, \eta_{A}, \phi, \eta_{B}\right)= & P\left(x_{A}^{\theta} ; \theta, \eta_{A}\right) \\
& \times P\left(x_{B}^{\phi} ; \phi, \eta_{B} \mid x_{A}^{\theta} ; \theta, \eta_{A}\right),
\end{aligned}
$$

where $P\left(x_{A}^{\theta} ; \theta, \eta_{A}\right)$ is the probability density of measurement outcomes $x_{A}^{\theta}$ on mode $\mathrm{A}$, and $P\left(x_{B}^{\phi} ; \phi, \eta_{B} \mid x_{A}^{\theta} ; \theta, \eta_{A}\right)$ is the conditional probability density of measurement outcomes of quadrature $x_{B}^{\phi}$ on mode $\mathrm{B}$ provided that a particular measurement outcome $x_{A}^{\theta}$ was obtained on mode $\mathrm{A}$.
Since mode $\mathrm{A}$ is in a thermal state, the probability $P\left(x_{A}^{\theta} ; \theta, \eta_{A}\right)$ does not depend on $\theta$, and all quadratures $x_{A}^{\theta}$ exhibit Gaussian distribution with zero mean and variance

$$
V_{A}=\frac{1}{2}\left[\eta_{A} \cosh (2 r)+1-\eta_{A}\right] .
$$

This formula accounts for imperfect detection with efficiency $\eta_{A}$, and $\frac{1}{2} \cosh (2 r)$ is the variance of quadratures of mode A of the pure two-mode squeezed vacuum state 77. Explicitly, the probability density reads

$$
P\left(x_{A}^{\theta} ; \theta, \eta_{A}\right)=\frac{1}{\sqrt{2 \pi V_{A}}} \exp \left[-\frac{\left(x_{A}^{\theta}\right)^{2}}{2 V_{A}}\right] .
$$

Homodyne detection of quadrature $x_{A}^{\theta}$ on mode A of the two-mode squeezed vacuum state $(7)$ prepares the other mode $\mathrm{B}$ in a coherently displaced squeezed state with squeezing ellipse rotated by angle $-\theta$, see Fig. 1(b). This rotation follows from the identity

$$
U_{A}(\theta) U_{B}(-\theta)|\xi\rangle=|\xi\rangle,
$$

where $U(\theta)=e^{-i n \theta}$ is a unitary phase shift operator. Measurement of a rotated quadrature $x_{A}^{\theta}$ on mode $\mathrm{A}$ is thus fully equivalent to measurement of quadrature $x_{A}$, followed by rotation of mode B by $-\theta$. The covariance matrix of the conditionally prepared state does not depend on the measurement outcome $x_{A}^{\theta}$, and the coherent displacement $d$ is linearly proportional to the measurement outcome.

Let $V_{-}$and $V_{+}$denote the variances of squeezed and anti-squeezed quadratures of the conditionally prepared state, and let $d$ denote the coherent displacement od the 
squeezed quadrature of this state. It follows from the above discussion that, without loss of generality, we can assume $\theta=0$ in our derivation of $V_{-}, V_{+}$, and $d$. It is convenient to collect the quadrature operators of modes $\mathrm{A}$ and $\mathrm{B}$ into a vector $z=\left(x_{A}, p_{A}, x_{B}, p_{B}\right)$ and define a two-mode covariance matrix $\gamma_{j k}=\left\langle\Delta z_{j} \Delta z_{k}+\Delta z_{k} \Delta z_{j}\right\rangle$, where $\Delta z_{j}=z_{j}-\left\langle z_{j}\right\rangle$. Covariance matrix of a two-mode squeezed vacuum state (7) whose mode A was transmitted through a lossy channel with transmittance $\eta_{A}$ reads,

$$
\gamma_{A B}=\left(\begin{array}{cccc}
2 V_{A} & 0 & K & 0 \\
0 & 2 V_{A} & 0 & -K \\
K & 0 & 2 V_{B} & 0 \\
0 & -K & 0 & 2 V_{B}
\end{array}\right)
$$

where $V_{B}=\frac{1}{2} \cosh (2 r)$ and $K=\sqrt{\eta_{A}} \sinh (2 r)$.

Since there are no correlations between the $x_{A}$ and $p_{B}$ quadratures, measurement of $x_{A}$ does not influence $p_{B}$, whose variance remains equal to $V_{B}$ and $\left\langle p_{B}\right\rangle=0$,

$$
V_{+}=\frac{1}{2} \cosh (2 r) \text {. }
$$

In contrast, the measurement of $x_{A}$ will reduce fluctuations of $x_{B}$ due to the correlations between $x_{A}$ and $x_{B}$. The resulting (conditional) variance $V_{-}$of $x_{B}$ can be calculated by minimizing the variance of $x_{B}-g x_{A}$ over a tunable gain $g$. The optimal gain reads $g_{\text {opt }}=K /\left(2 V_{A}\right)$, which yields

$$
V_{-}=\frac{1}{2} \frac{\eta_{A}+\left(1-\eta_{A}\right) \cosh (2 r)}{\eta_{A} \cosh (2 r)+1-\eta_{A}} .
$$

Moreover, the coherent displacement $d$ of the conditionally prepared state of mode $\mathrm{B}$ is given by $\left\langle x_{B}\right\rangle=g_{\mathrm{opt}} x_{A}$, which explicitly reads

$$
d=\frac{\sqrt{\eta_{A}} \sinh (2 r)}{\eta_{A} \cosh (2 r)+1-\eta_{A}} x_{A}
$$

The variances $V_{-}$and $V_{+}$of squeezed and antisqueezed quadratures of the probe single-mode state determine the effective detection efficiency $\eta_{A}$ of $\mathrm{HD}_{A}$ and the parameter $\lambda$ of the (virtual) two-mode squeezed vacuum state (7). By inverting formulas $(16$ ) and (17), we get

$$
\eta_{A}=\frac{2\left(V_{+}-V_{-}\right)}{\left(2 V_{+}-1\right)\left(2 V_{-}+1\right)},
$$

and

$$
\lambda=\sqrt{\frac{2 V_{+}-1}{2 V_{+}+1}}
$$

The effective detection efficiency $\eta_{A}>\frac{1}{2}$ if and only if the probe state is squeezed and $V_{-}<\frac{1}{2}$. This establishes single-mode squeezing as a valuable resource for continuous variable quantum process tomography. If the probe state is pure, $V_{+}=1 /\left(4 V_{-}\right)$, then $\eta_{A}=1$. If $V_{-}<\frac{1}{2}$ then the efficiency is a decreasing function of $V_{+}$and in the limit $V_{+} \rightarrow \infty$ we get $\eta_{A}=1 /\left(1+2 V_{-}\right)$. The coherent displacement 18 of mode $\mathrm{B}$ can be expressed in terms of the quadrature variances as follows,

$$
d=\sqrt{2\left(V_{+}-V_{-}\right)} \sqrt{\frac{2 V_{-}+1}{2 V_{+}+1}} x_{A} .
$$

Formula (11) together with the above results suggests that the joint quadrature distribution $P\left(x_{A}^{\theta}, x_{B}^{\phi} ; \theta, \eta_{A}, \phi, \eta_{B}\right)$ can be sampled as follows. Generate random $x_{A}^{\theta}$ drawn from the Gaussian distribution (13) and a random $\theta$ and $\phi$ drawn from a uniform distribution in the $[0,2 \pi]$ interval. Prepare a single-mode squeezed Gaussian state with variances $V_{-}$ and $V_{+}$and displacement $d$, rotated in phase space by $-\theta$, as illustrated in Fig. 1(b). Send this probe state through the quantum channel $\mathcal{E}$ and measure a rotated quadrature $x_{B}^{\phi}$ of the output state with a homodyne detector. Note that the squeezing properties of the input probe states do not depend on $x_{A}^{\theta}$ and $\theta$, hence a source producing squeezed states with a fixed amount of squeezing and anti-squeezing is sufficient.

The improvement achieved by squeezed probe states in comparison to coherent probe states comes at a cost of somewhat increased experimental difficulty. In particular, the variances $V_{+}$and $V_{-}$of the probe squeezed states need to be precisely characterized, which can be achieved by routine homodyne detection, and these parameters have to be kept constant during the whole tomographic measurement. Moreover, the orientation of the squeezing ellipse should be fully under control and tunable to any required angle $\theta$. Finally, the ability to coherently displace the squeezed state is also required, which can be achieved e.g. by mixing it with an auxiliary coherent beam on a highly unbalanced beam splitter 40.

\section{TOMOGRAPHY OF QUANTUM MEASUREMENTS}

The proposed method can be also adapted to tomographic characterization of quantum measurements 31 33, 41,44. Consider a detector D which can respond with $K$ different outcomes. Each outcome is associated with a POVM element $\Pi^{k}$ and the probability to observe an outcome $k$ for input state $\rho$ reads $p(k)=\operatorname{Tr}\left[\Pi^{k} \rho\right]$. Consider now an ancilla-assisted quantum detector tomography [43, 44], where the detector is probed with one part of a two-mode squeezed vacuum state, see Fig. 2(a). The conditionally prepared state of mode A corresponding to measurement outcome $k$ on mode B can be expressed as

$$
\rho^{k}=\left(1-\lambda^{2}\right) \sum_{m=0}^{\infty} \sum_{n=0}^{\infty} \lambda^{m+n} \Pi_{m, n}^{k}|n\rangle\langle m| .
$$

The state is not normalized and its trace is equal to probability of observing the outcome $k$,

$$
\operatorname{Tr}\left(\rho^{k}\right)=\left\langle\xi\left|I_{A} \otimes \Pi_{B}^{k}\right| \xi\right\rangle .
$$




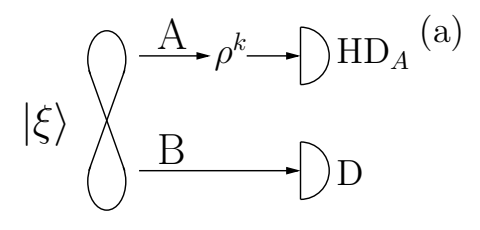

FIG. 2: (a) Ancilla-assisted quantum detector tomography 43 44. An unknown detector $\mathrm{D}$ is probed with one part of two-mode squeezed vacuum state $|\xi\rangle$. Information about POVM element $\Pi^{k}$ associated with measurement outcome $k$ of detector $D$ is imprinted into the corresponding conditional state $\rho^{k}$ of mode A, which can be characterized by homodyne tomography. (b) An equivalent scheme where the detector is probed with an ensemble of Gaussian squeezed states.

Formula 22 implies that the information about the POVM element $\Pi^{k}$ is imprinted into the conditional state $\rho^{k}$. In particular, we have

$$
\Pi_{m, n}^{k}=\lambda^{-(m+n)}\left(1-\lambda^{2}\right)^{-1} \rho_{n, m}^{k},
$$

in analogy with Eq. (8). The conditional states $\rho^{k}$ can be characterized by homodyne detection on mode A, which would provide sufficient data to reconstruct the density matrix elements $\rho_{m, n}^{k}$.

Similarly as for quantum operations, probing with one part of two-mode squeezed vacuum can be replaced by probing with single-mode squeezed states, see Fig. 2(b). Let $p\left(k \mid x_{A}^{\theta}, \theta\right)$ denote the probability of outcome $k$ for a probe state with displacement and rotation specified by parameters $x_{A}^{\theta}$ and $\theta$, respectively, c.f. Eqs. 16, 17, and (18). The statistics of homodyne measurements on $\rho^{k}$ is governed by $P\left(x_{A}^{\theta}, \theta, \eta_{A}\right) p\left(k \mid x_{A}^{\theta}, \theta\right)$, where $\eta_{A}$ is a function of the variances of squeezed and anti-squeezed quadratures of the probe squeezed state, see Eq. (19). The density matrix elements of $\rho^{k}$ can be obtained by averaging appropriate pattern functions over the quadrature statistics,

$$
\begin{aligned}
\rho_{m, n}^{k}= & \frac{1}{2 \pi} \int_{0}^{2 \pi} \int_{-\infty}^{\infty} P\left(x_{A}^{\theta}, \theta, \eta_{A}\right) p\left(k \mid x_{A}^{\theta}, \theta\right) \\
& \times f_{m, n}\left(x_{A}^{\theta}, \eta_{A}\right) e^{i(m-n) \theta} d x_{A}^{\theta} d \theta .
\end{aligned}
$$

The matrix elements of $\Pi^{k}$ can then be immediately obtained from Eq. (24), where the parameter $\lambda=\tanh r$ is determined by the variance of anti-squeezed quadrature $V_{+}$of the probe state, see Eq. 16.

\section{CONCLUSIONS}

In summary, we have proposed a procedure for tomographic characterization of continuous variable quantum operations which employs homodyne detection and single-mode squeezed probe states with a fixed degree of squeezing and anti-squeezing and a variable displacement and orientation of squeezing ellipse. We have shown that the elements of quantum process matrix $\chi$ in Fock basis can be estimated by averaging suitable pattern functions over the homodyne data. The pattern functions are well behaved provided that the probe state is squeezed and $V_{-}<\frac{1}{2}$. For the sake of simplicity, we have considered tomography of a single-mode operation $\mathcal{E}$. However, the method can be straightforwardly extended to multimode operations. For tomography of $\mathrm{N}$-mode operation, one would have to use $N$ independent single-mode squeezed states and measure each output mode with an independent homodyne detector. While we have focused on linear reconstruction procedure based on pattern function formalism, other methods of data processing would be also possible. For instance, one may utilize the widely employed maximum-likelihood estimation, or other approaches. Given its relative simplicity and practical feasibility, the present procedure is likely to find applications in the characterization of continuous variable quantum operations and measurements.

\section{Acknowledgments}

The research leading to these results has received funding from the EU FP7 under Grant Agreement No. 308803 (Project BRISQ2), co-financed by MŠMT ČR (7E13032).
[1] J. F. Poyatos, J. I. Cirac, P. Zoller, Phys. Rev. Lett. 78, 390 (1997).

[2] I.L. Chuang and M.A. Nielsen, J. Mod. Opt. 44, 2455 (1997).

[3] A. Luis, and L.L. Sánchez-Soto, Phys. Lett. A 261, 12 (1999).

[4] A. Luis, Phys. Rev. A 62, 054302 (2000).

[5] J. Fiurasek and Z. Hradil, Phys. Rev. A 63, 020101(R) (2001).

[6] U. Leonhardt, Measuring the Quantum State of Light (Cambridge University Press, Cambridge, 1997).

[7] M.G.A. Paris and J. Řeháček, Eds., Quantum State Es- timation, Lecture Notes in Physics Vol. 649 (Springer, Berlin, 2004).

[8] A. I. Lvovsky and M. G. Raymer, Rev. Mod. Phys. 81, 299 (2009).

[9] G.M. D'Ariano and P. Lo Presti, Phys. Rev. Lett. 86, 4195 (2001).

[10] W. Dür and J. I. Cirac, Phys. Rev. A 64, 012317 (2001).

[11] J.B. Altepeter, D. Branning, E. Jeffrey, T.C. Wei, P.G. Kwiat, R.T. Thew, J.L. O'Brien, M.A. Nielsen, and A.G. White, Phys. Rev. Lett. 90, 193601 (2003).

[12] F. De Martini, A. Mazzei, M. Ricci, G. M. D'Ariano, Phys. Rev. A 67, 062307 (2003). 
[13] M. Mohseni, A. T. Rezakhani, D. A. Lidar, Phys. Rev. A 77, 032322 (2008).

[14] M.-D. Choi, Linear Algebra Appl. 10, 285 (1975).

[15] A. Jamiolkowski, Rep. Math. Phys. 3, 275 (1972).

[16] M. A. Nielsen, E. Knill, R. Laflamme, Nature 396, 52 (1998).

[17] M. W. Mitchell, C. W. Ellenor, S. Schneider, and A. M. Steinberg, Phys. Rev. Lett. 91, 120402 (2003).

[18] J.L. O'Brien, G.J. Pryde, A. Gilchrist, D.F.V. James, N.K. Langford, T.C. Ralph, and A.G. White, Phys. Rev. Lett. 93, 080502 (2004).

[19] M. Riebe, K. Kim, P. Schindler, T. Monz, P. O. Schmidt, T. K. Körber, W. Hänsel, H. Häffner, C. F. Roos, and R. Blatt, Phys. Rev. Lett. 97, 220407 (2006).

[20] A. Černoch, J. Soubusta, L. Bartušková, M. Dušek, and J. Fiurášek, Phys. Rev. Lett. 100, 180501 (2008).

[21] A. M. Childs, I. L. Chuang, D. W. Leung, Phys. Rev. A 64, 012314 (2001).

[22] T. Baumgratz, D. Gross, M. Cramer, and M.B. Plenio, Phys. Rev. Lett. 111, 020401 (2013).

[23] M. Holzäpfel, T. Baumgratz, M. Cramer, and M.B. Plenio, Phys. Rev. A 91, 042129 (2015).

[24] D. Gross, Y.-K. Liu, S.T. Flammia, S. Becker, and J. Eisert, Phys. Rev. Lett. 105, 150401 (2010).

[25] A. Shabani, R.L. Kosut, M. Mohseni, H. Rabitz, M.A. Broome, M.P. Almeida, A. Fedrizzi, and A.G. White, Phys. Rev. Lett. 106, 100401 (2011).

[26] A.V. Rodionov, A. Veitia, R. Barends, J. Kelly, D. Sank, J. Wenner, J.M. Martinis, R.L. Kosut, and A.N. Korotkov, Phys. Rev. B 90, 144504 (2014).

[27] M. Lobino, D. Korystov, C. Kupchak, E. Figueroa, B.C. Sanders, and A.I. Lvovsky, Science 322, 563 (2008).

[28] S. Rahimi-Keshari, A. Scherer, A. Mann, A.T. Rezakhani, A.I. Lvovsky, and B.C. Sanders, New J. Phys. 13, 013006 (2011).

[29] A. Anis and A.I. Lvovsky, New J. Phys. 14105021
(2012).

[30] R. Kumar, E. Barrios, and C. Kupchak, and A.I. Lvovsky, Phys. Rev. Lett. 110, 130403 (2013).

[31] J.S. Lundeen, A. Feito, H. Coldenstrodt-Ronge, K.L. Pregnell, Ch. Silberhorn, T.C. Ralph, J. Eisert, M.B. Plenio, and I.A. Walmsley, Nature Phys. 5, 27 (2009).

[32] A. Feito, J.S. Lundeen, H. Coldenstrodt-Ronge, J. Eisert, M.B. Plenio, and I.A. Walmsley, New J. Phys. 11, 093038 (2009).

[33] V. DAuria, N. Lee, T. Amri, C. Fabre, and J. Laurat, Phys. Rev. Lett. 107, 050504 (2011).

[34] G. Brida, L. Ciavarella, I. P. Degiovanni, M. Genovese, L. Lolli, M. G. Mingolla, F. Piacentini, M. Rajteri, E. Taralli, and M. G. A. Paris, New J. Phys. 14, 085001 (2012).

[35] P.C. Humphreys, B.J. Metcalf, T. Gerrits, T. Hiemstra, A.E. Lita, J. Nunn, S.W. Nam, A. Datta, W.S. Kolthammer, and I.A. Walmsley, arXiv:1502.07649 (2015).

[36] J. R. Klauder, Phys. Rev. Lett. 16, 534 (1966).

[37] G. M. D'Ariano, U. Leonhardt and H. Paul, Phys. Rev. A 52, R1801 (1995).

[38] U. Leonhardt, M. Munroe, T. Kiss, Th. Richter, and M.G. Raymer, Opt. Commun. 127, 144 (1995).

[39] Th. Richter, Phys. Rev. A 61, 063819 (2000).

[40] A. Furusawa, J. L. Sørensen, S. L. Braunstein, C. A. Fuchs, H. J. Kimble, and E.S. Polzik, Science 282, 706 (1998).

[41] A. Luis and L. L. Sánchez-Soto, Phys. Rev. Lett. 83, 3573 (1999).

[42] J. Fiurášek, Phys. Rev. A 64, 024102 (2001).

[43] T. Amri, J. Laurat, and C. Fabre, Phys. Rev. Lett. 106, 020502 (2011).

[44] G. Brida, L. Ciavarella, I.P. Degiovanni, M. Genovese, A. Migdall, M.G. Mingolla, M.G.A. Paris, F. Piacentini, and S.V. Polyakov, Phys. Rev. Lett. 108, 253601 (2012). 